\title{
Cancer Care and COVID-19 Pandemic: Coping with a Double Challenge
}

\section{Firdous Barbhuiya}

Tata Institute of Social Sciences, Mumbai, India.

Asian Pac J Cancer Care, 5 (Suppl 1), 225-227

\section{Introduction}

The outbreak of Coronavirus disease 2019 (COVID-19), caused by SARS-CoV-2, has affected the lives of millions of people in more than 210 countries and territories around the globe. Since the diagnosis of the first COVID-19 case in December, 2019, a total of 7,495,828 coronavirus cases have been reported with $3,270,599$ infected and 419,804 death cases worldwide by $11^{\text {th }}$ June, 2020 [1]. As the pandemic escalates, emerging Europe, and America as the epicentre, countries struggle to contain the virus by adopting different health and socio-economic measures.

A huge surge in the COVID-19 cases has been apparent in South-east Asia, and the World Health Organisation (WHO) reported the presence of infection clusters in India [2]. The Ministry of Health and Family Welfare reports 286,579 confirmed cases and 8102 COVID-19 deaths in India by 11th June, 2020 [3]. With a rapid transmission rate and a huge number of infected cases, the pandemic poses an unprecedented challenge to the healthcare system. The mortality from other severe illnesses like cancer remains substantial, which needs to be significantly recognised. Among the different groups who are more vulnerable to COVID-19, cancer patients stand in the frontline whose bio-psychosocial health has been highly affected.

Cancer is the second leading cause of global mortality, accounting for around 9.6 million deaths in 2018 [4]. In India, it is the second most common disease responsible for maximum death, causing more than $10 \%$ of the annual mortality [5]. According to Bray et al, around 1.16 million cancer incidence, 2.25 million prevalence cases, and 0.78 million cancer mortalities were reported in India in 2018 [6]. Patients with cancer usually require uninterrupted medical care and are always advised not to delay or discontinue their treatment. In the midst of the pandemic, it has become a huge challenge for the patients, doctors, and caregivers to continue treatment by weighing risks of COVID-19 morbidity and mortality against the benefit of
Submission Date: 06/11/2020Ａcceptance Date: 08/15/2020

cancer treatment.

\section{Oncological Determinants of COVID-19}

Cancer patients are more susceptible to develop COVID-19 infection than those without. It is because of their underlying illness status and systemic immunosuppressive state caused by the malignancy, anti-cancer treatments viz. radiotherapy, chemotherapy, targeted therapy, and immunotherapy, etc. and the side-effects of these treatments. The WHO-China Joint Report estimates cancer patients at a 2-fold risk of developing COVID-19 than the general population [7].

Liang et al also reported a significantly higher incidence and risk of severe complications among cancer patients from the virus including intensive care unit (ICU) admission, invasive ventilation, or even death [8]. Although the model included adjustments for age, sex, and comorbidities, the interpretation of the findings was limited by a small sample size of 18 cancer patients. Further, studying clinical characteristics of COVID-19, Guan et al revealed $6.1 \%$ of the cancer patients required ICU care or mechanical ventilation [9]. Although it was significant to the situation of China and Italy, it is yet unclear how the virus will behave in India.

In addition to the fact of being more prone to SARS-CoV-2 infection, cancer patients continue to face difficulties in accessing the necessary medical care. The pandemic has raised two important issues associated with cancer patients' safety. First, cancer patients have to visit clinics or hospitals regularly and thereby exposing themselves to COVID-19 infection. Second, cancer-specific treatments themselves can expose the patients to more detrimental effects by weakening their immune system. The fundamental question arises: should the patient be exposed to SARS-CoV-2 for receiving cancer treatments? Should the adjuvant chemotherapy or elective surgery for patients be postponed? Should cancer

Corresponding Author:

Mr. Firdous Barbhuiya

Tata Institute of Social Sciences, Mumbai, India.

Email: firdousbarbhuiya@gmail.com 
patients and survivours be advised for stronger personal protection? How to deal with a possible delay in clinical trials if enforced quarantine or social distancing measures obscures the hospital attendance or continuity in cancer care? The available evidence for a precise answer to these questions is very limited, in the absence of sufficient studies on cancer patients and COVID-19. Besides, in view of the coronavirus, there is no guideline for cancer care concerning the kinds of cancer, treatment types, and the demography of the patients.

A meta-analysis reveals that COVID-19 has a higher prevalence among cancer patients and survivours than the general population [10]. The study also reported a pooled prevalence of COVID-19 of 2-3\% while analysing different studies with different sample sizes. The study reveals that evidence on the association between cancer and COVID-19 remains inconclusive [10]. However, clear evidence exists that old age, higher levels of comorbidities and weak immunity are associated with more severe COVID-19 effects. This consideration is highly relevant to cancer patients. This implies that cancer patients need serious attention particularly those undergoing bone marrow or stem-cell transplantation, those with hematologic malignancies, and those receiving active treatments, considering the higher risk.

To contain the spread of COVID-19, different health and socio-economic measures have been adopted and people are advised to stay at home and visit outside only in pressing need. Consequently, enforced quarantine, nationwide lockdown, and delayed clinical trials have complicated the appearance in the hospital for appointments and continuity in the care of advanced staged cancer patients with severe health complications. Adverse effects may be experienced by patients receiving immune checkpoint inhibitors (viz. severe myocarditis and pneumonitis), making the proper diagnosis and treatment challenging. In balancing cancer care during the pandemic, careful consideration needs to be given to both patient-level and system-level risks of COVID-19. Patient-level factors (viz. age, place of residence, comorbidities, stage of cancer, and health practices) and patient's preferences regarding risks and benefits of cancer treatment during the pandemic need to be considered in the light of system-level factors (viz. healthcare resources, clinics, personnel, transportation, availability, and accessibility of services).

\section{Restructuring Cancer Care during COVID-19 Outbreak}

The pandemic has blown cancer care in a very predicament situation because neither the cancer treatment should be delayed nor the patients should be exposed to COVID-19 risks. Also, the patients and doctors have no choice but to take the pandemic into account in the treatment decisions. Besides, the more difficult decisions are clinical decisions regarding the treatment delay. Clinical decisions should consider individual factors viz. stage of cancer, type of treatments undergoing/ need to undergo, and the risk of recurrence if treatments are delayed.

Cancer clinics and hospitals need to adopt altered standardised care to prevent anti-cancer treatment-induced complications of COVID-19 and minimise hospital visits and hospitalisation. Considering the current crisis, elective surgeries of cancer patients can be postponed after individualised risk/ benefit assessment [11]. Besides currently, no evidence exists regarding holding chemotherapy or immunotherapy or delaying radiotherapy or adjuvant therapy [10]. Although there may be cases in which delaying adjuvant chemotherapy may be appropriate. Further, a few studies suggest that patients treated with chemotherapy are at risk of severe complications of influenza [12], but it is uncertain how this might derive to COVID-19. Also, many experts express that delaying chemotherapy because of the fear of COVID-19 would be a miscalculation of risks and benefits, as it is evident that delayed administration of chemotherapy is usually less effective.

Some kinds of treatment need to be paused while monitoring the course of COVID-19. Treatment delays during the pandemic may bring a due strain on the healthcare system due to travel restrictions, lack of sufficient workforce, affect on the drug supply chain, lack of access to hospital care, and others. Further, planned allogeneic stem-cell transplantation can be postponed considering COVID-19 outbreak especially when the disease is under control [11]. On the other hand, many patients with aggressive malignancy cannot afford to delay their curative treatments in the form of stem-cell transplantation and cellular immunotherapies. Besides, solid tumour patients receiving adjuvant therapy with curative intent are advised not to discontinue or delay the treatment during the pandemic. Delayed treatment for metastatic patients might lead to degraded performance status and poor chances of survival. Therefore, clinical decisions should be individualised considering different patient-level factors and logistical challenges in the system surfaced during the pandemic.

\section{Looking Forward}

The COVID-19 pandemic has brought unprecedented challenges before the healthcare system. The impending trajectory of the pandemic is very uncertain, and it instills us for better preparedness to face widespread impact. With higher vulnerability, the foremost challenge for cancer patients is to receive essential medical services, in a critical time when emergency departments and ICUs are close to breaking point and the medical resources are diverted to tackle the crisis. In view of the impact of COVID-19, there are continued efforts on flattening the curve and these should not compromise the health and well-being of patients with cancer. The pandemic situation is dynamic, and the policies, recommendations, and actions should address the topical crisis and evolving issues mounting from COVID-19.

In the presence of limited facts and findings, cancer patients should be considered at high risk for COVID-19. The patients should be taken care of against the virus by protective health behaviour including physical and social distancing measures, respiratory barriers, personal hygienic habits, and speedy treatment if infected by 
COVID-19. With respect to cancer treatment, some kinds of treatment could be delayed by assessing their risks and benefits in the light of COVID-19. Besides, hospital visits and continuity in care for many advanced staged cancer patients are very exigent and far from elective. The treatment decisions and choices cannot be one-size-fits-all, rather should be individualised. The impact of deferral and other associated outcomes needs to be weighed considering different patient-level and system-level priorities.

Further, health professionals engaged in cancer care have a critical role in communicating the patients about appropriate medical care and information regarding practice modifications in view of the outbreak. Considering the serious medical and emotional needs, the Government needs to issue a scientific guideline regarding the management of cancer patients and their care in the background of the COVID-19 outbreak. Throughout the pandemic, supporting the patients, addressing their emotional well-being, and providing adequate psycho-social support would be much more essential than ever. Thus, it is high time for a collective effort to think and act both nationally and locally to ensure that the interventions for the patients are relevant, appropriate, and healthy.

\section{References}

1. Worldometer. 2020. Available from: https://www. worldometers.info/coronavirus/? [Last accessed on 2020 June 11].

2. World Health Organization. Coronavirus disease 2019 (COVID-19) Situation Report - 97; 2020. Accessed from: https://www.who.int/docs/default-source/coronaviruse/ situation-reports/20200426-sitrep-97-covid-19. pdf?sfvrsn=d1c3e800_6 [Last accessed on 2020 April 28].

3. Ministry of Health and Family Welfare. Government of India. Accessed from https://www.mohfw.gov.in/ Accessed on 2020 June 11.

4. World Health Organization. Cancer Key Facts. Geneva: World Health Organization; 2018. Available from: https://www. who.int/news-room/fact-sheets/detail/cancer [Last accessed on 2020 April 28].

5. Ali I, Wani W, Saleem K. Cancer Scenario in India with Future Perspectives. Cancer Therapy. 2011;8:56-70 . http:// licgreaternoida.com/data/documents/CancerTherapy2011. pdf. Accessed on November 20, 2020.

6. Bray F, Ferlay J, Soerjomataram I, Siegel RL, Torre LA, Jemal A. Global cancer statistics 2018: GLOBOCAN estimates of incidence and mortality worldwide for 36 cancers in 185 countries. CA: A Cancer Journal for Clinicians. 2018 09 12;68(6):394-424. https://doi.org/10.3322/caac.21492

7. World Health Organization. Report of the WHO-China Joint Mission on COVID-19, 16-24 February 2020. Geneva: World Health Organization. 2020.

8. Liang W, Guan W, Chen R, Wang W, Li J, Xu K, Li C, Ai Q, Lu W, Liang H, Li S, He J. Cancer patients in SARSCoV-2 infection: a nationwide analysis in China. The Lancet Oncology. 2020 03;21(3):335-337. https://doi.org/10.1016/ s1470-2045(20)30096-6

9. Guan W, Ni Z, Hu Y, Liang W, Ou C, He J, Liu L, Shan H, Lei C, Hui DS, Du B, Li L, Zeng G, Yuen K, Chen R, Tang C, Wang T, Chen P, Xiang J, Li S, Wang J, Liang Z, Peng
Y, Wei L, Liu Y, Hu Y, Peng P, Wang J, Liu J, Chen Z, Li G, Zheng Z, Qiu S, Luo J, Ye C, Zhu S, Zhong N. Clinical Characteristics of Coronavirus Disease 2019 in China. New England Journal of Medicine. 202004 30;382(18):17081720. https://doi.org/10.1056/nejmoa2002032

10. Desai A, Sachdeva S, Parekh T, Desai R. COVID-19 and Cancer: Lessons From a Pooled Meta-Analysis. JCO Global Oncology. 2020 04;(6):557-559. https://doi.org/10.1200/ go. 20.00097

11. Shankar A, Saini D, Roy S, Mosavi Jarrahi A, Chakraborty A, Bharti SJ, Taghizadeh-Hesary F. Cancer Care Delivery Challenges Amidst Coronavirus Disease - 19 (COVID-19) Outbreak: Specific Precautions for Cancer Patients and Cancer Care Providers to Prevent Spread. Asian Pacific Journal of Cancer Prevention. 202003 01;21(3):569-573. https://doi.org/10.31557/apjcp.2020.21.3.569

12. van de Haar J, Hoes LR, Coles CE, Seamon K, Fröhling S, Jäger D, Valenza F, de Braud F, De Petris L, Bergh J, Ernberg I, Besse B, Barlesi F, Garralda E, Piris-Giménez A, Baumann M, Apolone G, Soria JC, Tabernero J, Caldas C, Voest EE. Caring for patients with cancer in the COVID-19 era. Nature Medicine. 202004 16;26(5):665-671. https://doi. org/10.1038/s41591-020-0874-8

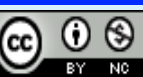

This work is licensed under a Creative Commons AttributionNon Commercial 4.0 International License. 